\title{
(Re)significando a doença celíaca na infância e (re)conhecendo as abordagens gerais através de uma revisão narrativa
}

\author{
(Re)meaning celiac disease in childhood and (re)knowing the general approaches through \\ a narrative review
}

\author{
$(\mathrm{Re})$ significando la enfermedad celíaca en la infancia y (re)conociendo los enfoques \\ generales a través de una revisión narrativa
}

Caroline Silva de Araujo Lima ${ }^{1 *}$, Ana Luisa Cabral dos Santos ${ }^{2}$, Anderson Poubel Batista ${ }^{3}$, Emmily Matias Pereira ${ }^{4}$, Gabriela Campos de Almeida ${ }^{5}$, Isah Maria Santos Pereira ${ }^{6}$, Leticia Stefanelli Potsch $^{4}$, Raphaela Henriques Ferreira ${ }^{3}$, Renata Guarçoni Bertoldi ${ }^{3}$, Nestor Augusto Avelino Leite ${ }^{7}$.

\section{RESUMO}

Objetivo: Evidenciar as manifestações clínicas da doença celíaca na infância e a importância do conhecimento da patologia vigente nessa faixa etária, possibilitando propostas de intervenção a fim de promover melhoria na qualidade de vida para os portadores da doença. Revisão bibliográfica: A doença celíaca pode se apresentar sob três formas: clássica, não clássica e assintomática. A forma clássica é a mais frequente e envolve sintomas como diarreia e distensão abdominal, normalmente detectada nos primeiros anos de vida e mais prevalente no sexo feminino. O diagnóstico da doença celíaca é feito através de métodos como prova de absorção da D-xilose, dosagem de gordura nas fezes, dosagem de anticorpo antigliadina e biópsia de intestino delgado. O tratamento mais indicado para a patologia é a restrição do glúten da dieta, que é o principal causador da inflamação. Considerações finais: A doença celíaca é uma enteropatia crônica, prevalente em esfera mundial. Apresenta-se com grande frequência na infância e, se diagnosticada e tratada precocemente, favorece o bem-estar da criança, e adequado crescimento e desenvolvimento.

Palavras-chave: Alimentação, Doença celíaca, Infância.

\begin{abstract}
Objective: To highlight the clinical manifestations of celiac disease in childhood and the importance of the knowledge of the pathology in this age group, enabling proposals for intervention and consequently a better quality of life for those with the disease. Bibliografic review: Celiac disease may present in three forms: classic, non-classic and asymptomatic. The classic form is the most frequent, where it involves symptoms such as diarrhea and abdominal distension, in which it is detected in the first years of life, being more prevalent in women. The diagnosis of celiac disease is made through methods such as D-xilosis absorption test, stool fat dosage, antigliadin antibody dosage and small intestine biopsy. The most indicated treatment for the pathology is the restriction of the gluten in the diet, which is the main cause of the inflammation, making the patient have a restrictive diet for a more satisfactory life. Final considerations: It is concluded that celiac disease is a chronic enteropathy prevalent in the world, with great frequency in childhood, and if diagnosed early, the child has better well-being throughout its growth and development.
\end{abstract}

Keywords: Food, Celiac disease, Childhood.

\section{RESUMEN}

Objetivo: Destacar las manifestaciones clínicas de la enfermedad celíaca en la infancia y la importancia del conocimiento de la patología en este grupo de edad, posibilitando propuestas de intervención y consecuentemente una mejor calidad de vida de los enfermos. Revisión bibliográfica: La enfermedad celíaca puede presentarse de tres formas: clásica, no clásica y asintomática. La forma clásica es la más

\footnotetext{
${ }^{1}$ Faculdade Dinâmica Vale do Piranga (FADIP), Ponte Nova - MG. *E-mail: carolinearaujo689@gmail.com

2 Universidade do Grande Rio (UNIGRANRIO), Duque de Caxias - RJ.

${ }^{3}$ Faculdade Metropolitana São Carlos (FAMESC), Bom Jesus do Itabapoana - RJ.

4 Universidade Vila Velha (UVV), Vila Velha - ES.

${ }^{5}$ Faculdade da Saúde e Ecologia Humana (FASEH), Vespasiano - MG.

${ }^{6}$ Faculdade Santo Agostinho (FASA), Itabuna - BA.

${ }^{7}$ Faculdade de Medicina de Campos, Campos dos Goytacazes - RJ.
} 
frecuente, en la que presenta síntomas como diarrea y distensión abdominal, en la que se detecta en los primeros años de vida, siendo más frecuente en las mujeres. El diagnóstico de la enfermedad celíaca se realiza mediante métodos como la prueba de absorción de la D-xilosis, la dosis de grasa en las heces, la dosis de anticuerpos antigliadina y la biopsia del intestino delgado. El tratamiento más indicado para la patología es la restricción del gluten en la dieta, que es la principal causa de la inflamación, lo que hace que el paciente tenga una dieta restrictiva para una vida más satisfactoria. Consideraciones finales: Se concluye que la enfermedad celíaca es una enteropatía crónica prevalente en el mundo, con gran frecuencia en la infancia, y si se diagnostica a tiempo, el niño tiene un mejor bienestar a lo largo de su crecimiento y desarrollo.

Palabras clave: Alimentación, Enfermedad celíaca, Infancia.

\section{INTRODUÇÃO}

A doença celíaca (DC) é uma doença crônica inflamatória que afeta indivíduos suscetíveis geneticamente e ocorre em decorrência à intolerância persistente ao glúten. O glúten, por sua vez, é o nome dado a um conjunto de proteínas presentes em alguns grãos como trigo, centeio, aveia, cevada e outros alimentos. $\mathrm{Na}$ DC, o indivíduo desenvolve uma enteropatia que se caracteriza pela atrofia parcial ou total das vilosidades da mucosa do intestino delgado, hiperplasia de criptas e aumento de linfócitos intraepiteliais após a exposição ao glúten (CONCEIÇÃO DD, et al., 2020).

É uma patologia de elevada prevalência mundial que acomete aproximadamente 1:100-300 pessoas no mundo. No Brasil, a prevalência é de, aproximadamente, $0,3 \%$ da população e estima-se que existam 300 mil brasileiros portadores da doença celíaca, com maior incidência na região sudeste (CAMPOS CP, et al., 2018). A média de idade do diagnóstico é $8,5 \pm 4,5$ anos, $69 \%$ são do sexo feminino, com maior gravidade quando diagnosticado em gestantes (OLIVEIRA GN, et al., 2018). Os estudos têm revelado ainda que esta patologia é mais frequente do que se acreditava, tendo a subnotificação de casos como uma característica. Essa subnotificação ocorre, principalmente, em consequência à desinformação e dificuldade de se estabelecer o diagnóstico correto (BRASIL, 2015).

A DC pode se apresentar sob três formas: clássica, não clássica e assintomática. A forma clássica é a mais frequente, presente em cerca de 59,7\% dos casos. Esta é detectada nos primeiros anos de vida e caracteriza-se por diarreia crônica, vômitos, anorexia, distensão abdominal, irritabilidade e déficit de crescimento nas crianças. Já a forma não clássica corresponde a cerca de $25,2 \%$ e manifesta-se mais tarde (61,1\% em maiores de 14 anos), sendo comum a ocorrência de baixa estatura, anemia por deficiência de ferro refratária à ferroterapia oral, hipoplasia do esmalte dentário e constipação intestinal. A forma assintomática deve ser investigada quando há história familiar de primeiro grau positiva para a doença (SDEPANIAN VL, et al., 2001).

Deve ser enfatizado que a idade de início dessa enteropatia pode influenciar na manifestação clínica da DC. As principais manifestações clínicas em crianças mais jovens são diarreia $(63,3 \%)$ e distensão abdominal $(59,1 \%)$, características da forma clássica de apresentação da doença. Por outro lado, a dor abdominal $(75,8 \%)$ predomina em crianças maiores de 5 anos. É imprescindível reconhecer que, no momento do diagnóstico da doença celíaca, várias outras condições podem estar associadas, como: diabetes mellitus tipo 1, deficiência de imunoglobulina $\mathrm{A}(\lg \mathrm{A})$, hipotireoidismo, distúrbios do neurodesenvolvimento e distúrbios psicológicos (OLIVEIRA GN, et al., 2018).

Além da sintomatologia característica, o paciente diagnosticado com a DC enfrenta grandes mudanças, principalmente quando ainda criança ou adolescente, haja vista a necessidade da modificação de condutas alimentares diárias estabelecidas pelos protocolos de tratamento desta doença. Por isso é de suma importância ressaltar que, apesar de pouquíssimos estudos, podemos observar nos pacientes celíacos sentimentos como vergonha, isolamento e objeções nas relações sociais e em atividades cotidianas (OLSSON C, et al., 2008).

Outra questão que deve ser abordada com o paciente portador da DC é sobre a existência de consequências imunológicas e metabólicas relacionadas a ingestão contínua de glúten, como: osteoporose, deficiência de ferro, calcificações cerebrais, distúrbios do comportamento e malignidade (DIAS JA, 2017). Por esse motivo, o tratamento consiste em uma dieta restritiva, em que se retira o glúten da alimentação do paciente durante toda a vida, evitando assim alterações físicas, sociais e mentais (OLIVEIRA GN, et al., 2018). 
Diante do exposto, o objetivo do presente trabalho é evidenciar as manifestações clínicas da doença celíaca na infância, ampliando-se assim o reconhecimento desta patologia, o que resultará em uma intervenção mais rápida e, consequentemente, em melhoria da qualidade de vida para os portadores dessa enteropatia crônica.

\section{REVISÃO BIBLIOGRÁFICA}

\section{Manifestações clínicas}

A doença celíaca (DC) é a intolerância alimentar mais prevalente na população ocidental, em decorrência da elevada presença de insumos alimentícios contendo glúten. Pão, bolo, macarrão, cereais e cerveja são alguns exemplos. Glúten é uma palavra derivada do latim gluten, que significa "cola" e é composto de duas proteínas principais, denominadas gliadina e glutenina (PAULINO R, 2017).

Segundo Paulino R (2017), o mecanismo pelo qual o glúten exerce função tóxica ainda não foi totalmente elucidado. Entretanto, foi observado que pacientes com tal doença possuem antígenos de histocompatibilidade HLA, sendo HLA DQ2 e HLA DQ8 os mais comuns. Esses antígenos, por sua vez, interagem com o glúten e causam resposta imunológica anormal, com liberação de citocinas por células $\mathrm{T}$, resultando em lesões severas na mucosa intestinal.

Verifica-se ainda que a patologia apresenta um importante componente genético em sua fisiopatologia, sendo mais susceptível em indivíduos com parentes de primeiro grau celíacos. De acordo com estudo realizado por Cavinatto J (2017), o risco de induzir DC pela dieta contendo glúten, diz respeito exclusivamente a pessoas que apresentam pelo menos um dos alelos (HLA-DQ2/DQ8), que são predisponentes para o desenvolvimento da doença. Isto aplica-se a $30 \%$ a $40 \%$ da população em geral e a $75 \%$ a $80 \%$ das pessoas que tem um dos pais ou irmãos com DC. Dessa forma, faz-se necessário o acompanhamento adequado de crianças com histórico familiar, a fim de evitar complicações.

Além disso, fatores ambientais também podem contribuir para o desenvolvimento da enfermidade, como amamentação, idade de introdução do glúten na dieta da criança, ocorrência de certas infecções e microbiota intestinal. Estudos apontaram que o aleitamento materno pode produzir algum efeito protetor contra a DC, ao contrário da idade de introdução do glúten, que não apresentou influência direta no aumento do risco patológico (CAVINATTO J, 2017).

De acordo com o quadro clínico, a DC cursa com uma ampla variedade de sinais e sintomas. A partir do quadro clínico, esta patologia é classificada como: DC clássica, constituída por um quadro de má absorção com início nos primeiros anos de vida; DC não clássica, que se manifesta mais tardiamente, sendo geralmente mono ou oligossintomática e com presença predominante de manifestações extra intestinais, e assintomática. Além disso, certos indivíduos que apresentam risco de desenvolver DC futuramente são descritos como pertencentes ao grupo de DC potencial, normalmente por apresentarem histórico familiar associado (OLIVEIRA GN, et al., 2018).

$\mathrm{Na}$ forma clássica da doença, a dor abdominal e a diarreia crônica são os sintomas mais relatados. Tais pacientes costumam apresentar também distensão abdominal, esteatorreia, constipação, vômitos, emagrecimento e déficit de crescimento. Além disso, a enxaqueca foi a principal condição neurológica e a ansiedade o distúrbio psicológico mais comum. Outros sintomas menos frequentes são vômitos, náuseas, falta de apetite, fezes com sangue ou muco, irritabilidade e letargia (OLIVEIRA GN, et al., 2018). Em um estudo realizado por Isikay S (2015), observou-se que cerca de 10-22\% dos pacientes com doença celíaca apresentaram sintomas neurológicos além da enxaqueca, como ataxia, disfunção vestibular e convulsões. Foi proposto que a autoimunidade resultante do mimetismo molecular entre a gliadina e as proteínas do sistema nervoso pode desempenhar importante papel nas manifestações neurológicas da doença celíaca.

Além disso, o diagnóstico de DC em crianças com mais de cinco anos foi mais prevalente em relação à doença em crianças mais novas, o que comprova um atraso no reconhecimento dos casos que cursam com quadros atípicos e subclínicos. Ademais, estudos revelaram que a maioria dos pacientes permanece sem diagnóstico ou demora anos para ser estabelecida a presença de DC (OLIVEIRA GN, et al., 2018). 
Por ser uma intolerância permanente ao glúten, é imprescindível que os pacientes sejam diagnosticados o quanto antes, a fim de estabelecer o tratamento adequado e evitar possíveis complicações que a doença pode acarretar a longo prazo, como hipoplasia de esmalte dentário, artralgia ou artrite, distúrbios de comportamento, epilepsia associada a calcificações cerebrais e malignidades (DIAS JA, 2017).

A atrofia das vilosidades da mucosa do intestino delgado, decorrentes de lesões teciduais pela interação da gliadina com antígeno de histocompatibilidade HLA, é responsável pela má absorção de elementos essenciais, como ferro, ácido fólico e vitamina $B 12$, cálcio e vitamina $D$, gerando anemia ferropriva refratária à ferroterapia oral, anemia megaloblástica e osteoporose antes da menopausa, respectivamente. A baixa densidade mineral óssea pode acarretar em fragilidade óssea e aumento do risco de fraturas. Além disso, a má absorção de zinco, selênio e ácido fólico pode levar à infertilidade, abortos de repetição e prematuridade (IGARSABA L, 2019).

Outra manifestação clínica frequente da DC é a dermatite herpetiforme. Esta se manifesta na forma de erupção cutânea caracterizada pela presença crônica de bolhas, prurido intenso e sensação de queimação em pápulas eritematosas e placas urticariformes, acompanhada por vesículas (IGARSABA L, 2019).

O diagnóstico tardio da DC aumenta o risco de ocorrência de doenças associadas, sendo o diabetes mellitus tipo 1 (DM1) o distúrbio mais frequente, além de deficiência de IgA e hipotireoidismo. Esses distúrbios comprovam a base imunológica da etiopatogenia da DC, encontrada também nessas outras doenças. Conforme evidenciado por Damasceno J (2017), a prevalência de DC entre portadores de DM1 tem sido estimada em cerca de 4,0\% com variação entre 0,1 e 10,4\%, superando, em muito, a prevalência da população geral (entre 0,5 e 1,0\%). Além disso, pacientes com DC associada à DM1 possuem maior risco de evoluir para complicações, como retinopatia e nefropatia. Por esse motivo, é importante que pacientes diabéticos sejam rastreados quanto à $\mathrm{DC}$, pois na maioria dos casos o curso desta é assintomático (IGARSABA L, 2019).

A DC afeta a vida física, mental e social, bem como a qualidade de vida relacionada à saúde das crianças. Dessa forma, foi verificado que pelo menos um diagnóstico psiquiátrico estava presente em metade dos casos de portadores de DC, sendo o transtorno depressivo maior o distúrbio mais frequente. Além disso, a má absorção característica da doença e a baixa adesão à dieta sem glúten (GFD) promove deficiência de nutrientes como o triptofano, podendo causar um estado hiposserotonérgico no sistema nervoso central (SEVINÇ E, et al., 2017).

Crianças com diagnóstico de DC relataram sentimentos de isolamento e baixa autoestima, bem como dificuldades nas relações sociais e em atividades cotidianas, como a escolha de alimentos e o planejamento de viagens, em decorrência das sucessivas restrições impostas pela dieta isenta de glúten (BRANCAGLIONI $B C A, 2016)$. Para evitar isso, é necessário que crianças e adolescentes sejam conscientizados em relação à compreensão da patologia, adaptando-se à nova dieta exigida. Ao longo dos anos, torna-se evidente a produção e o desenvolvimento de versões de produtos alimentícios sem glúten, a fim de atender o público com privação a esse tipo de substância.

\section{Diferença entre adultos e crianças}

Historicamente, a DC foi considerada rara e predominantemente pediátrica. Atualmente, este cenário mudou, especialmente devido ao desenvolvimento de mais testes sorológicos sensíveis e específicos para diagnóstico da doença (CONCEIÇÃO DS, et al., 2020). Segundo Labrada OB, et al. (2020), cada vez mais casos estão sendo diagnosticados em pacientes adultos: às vezes são formas silenciosas ou inadvertidas durante a infância, mas outras vezes, essas são manifestações que realmente se iniciam na idade adulta. Por este motivo, muitos autores estão começando a considerar a doença celíaca uma doença desta época da vida também.

Como descrito, a doença celíaca pode se manifestar em qualquer idade e apresenta-se em duas formas. A forma não clássica, atípica ou subclínica da DC manifesta-se mais tardiamente, com quadro mono ou paucissintomático. Os pacientes deste grupo podem apresentar manifestações isoladas, como: baixa estatura, anemia por deficiência de ferro refratária à ferroterapia oral, hipoplasia do esmalte dentário, constipação intestinal, osteoporose, esterilidade, artralgia ou artrite e epilepsia associada à calcificação 
intracraniana (SDEPANIAN VL, et al., 2001). Enquanto a forma clássica ou típica, apresenta sorologia positiva específica e biópsia compatível com sinais e sintomas clássicos, tais como má absorção, diarreia crônica, anorexia, distensão abdominal, perda de massa muscular, nádegas achatadas, esteatorreia, edema de hipoalbuminemia, flatulência, fraqueza e irritabilidade (CONCEIÇÃO DS, et al., 2020).

Ao contrário das formas infantis clássicas, é incomum os casos diagnosticados em adultos com diarreia e sintomas de má absorção devido à atrofia das vilosidades intestinais pois, ao contrário, predominam os sintomas atípicos, entre estes, a ocorrência de anemia e deficiência de ferro (LABRADA OB, et al., 2020). Observa-se nos últimos anos uma mudança drástica na forma de apresentação da DC, com aumento de apresentações oligossintomáticas e até atípicas desta doença. A prevalência em adultos atinge $0,48 \%$, e aumenta para $4,5 \%$ na população de alto risco, conforme parentes de primeiro grau de pacientes com DC (LABRADA OB, et al., 2020).

As manifestações clínicas da $D C$ têm um amplo espectro que vai desde a síndrome de má absorção à doença assintomática e não é observada uma relação clara entre o quadro clínico e o grau de atrofia das vilosidades. A extensão do envolvimento intestinal também não está relacionada à gravidade da apresentação clínica. Reconheceu ainda a estreita relação entre DC e doenças autoimunes e endócrinas, de modo que o diabetes mellitus tipo 1 se destaca, com uma prevalência de DC de $3-10 \%$ em crianças e $2-5 \%$ em adultos (LABRADA OB, et al., 2020).

A doença celíaca tem impactos substanciais no psicológico, nas relações familiares e sociais dos pacientes diagnosticados, exigindo uma visão clínica biopsicossocial para melhor adesão ao tratamento e a qualidade de vida do paciente. Destes três pontos, há mudanças no estado emocional dos pacientes entrevistados, conflitos nas relações familiares e dificuldades sociais relacionamentos devido à dieta sem glúten. Por outro lado, o apoio da família e amigos provou ser um facilitador importante que teve um impacto significativo na adaptação dos pacientes, mostrando que um maior conhecimento de fatores biopsicossociais pode contribuir para uma melhora significativa na saúde geral e na qualidade de vida dos celíacos (CONCEIÇÃO DS, et al., 2020).

\section{Diagnóstico}

Até a década de 1950, a DC era um diagnóstico clínico baseado em observações focadas em características de má absorção. Atualmente, o primeiro passo para detectar se um paciente tem DC é obter sorologias (WATKINS RN e ZAWAHIR SM, 2017). Observou-se também que mães que dão à luz filhos com doença celíaca têm perfis de citocinas séricas de gravidez diferentes das mães de crianças saudáveis, sugerindo que o ambiente pré-natal pode ter um impacto no status de autoimunidade da prole (AGARDH DN, et al., 2017).

No que se refere ao estabelecimento do diagnóstico da doença celíaca, alguns métodos utilizados são: prova de absorção da D-xilose, dosagem de gordura nas fezes, dosagem de anticorpo antigliadina e biópsia de intestino delgado (NETO US, et al, 2001). Os anticorpos antigliadina foram desenvolvidos pela primeira vez na década de 1980, mas mostraram uma sensibilidade e especificidade entre $80 \%$ e $90 \%$. Já o anticorpo endomisial (EMA) foi desenvolvido em meados da década de 1980, que foi considerado altamente preciso, mas difícil de padronizar (WATKINS RN e ZAWAHIR SM, 2017).

Contudo, para a obter de forma definitiva o diagnóstico é válido realizar endoscopia digestiva alta com biópsia do intestino delgado para a análise no exame histopatológico, considerado o padrão-ouro para o diagnóstico. Dessa forma, a biópsia precisa apresentar pelo menos quatro fragmentos, incluindo amostra do bulbo e também as porções mais distais do duodeno (NATIONAL INSTITUTES OF HEALTH CONSENSUS DEVELOPMENT CONFERENCE STATEMENT ON CELIAC DISEASE, 2004; LUDVIGSSON JF, et al., 2014).

Nota-se que os marcadores sorológicos são fundamentais para: (i) a identificação dos indivíduos que necessitam ser submetidos à biópsia de intestino delgado e (ii) para o acompanhamento do paciente portador de doença celíaca. Sendo assim, vale recordar novamente, que os principais testes sorológicos para detectar a intolerância ao glúten são: (i) o anticorpo antigliadina, (ii) o anticorpo antiendomísio e o (iii) anticorpo anti transglutaminase (TTG) (NATIONAL INSTITUTES OF HEALTH CONSENSUS DEVELOPMENT CONFERENCE STATEMENT ON CELIAC DISEASE, 2004; LUDVIGSSON JF, et al., 2014). 
Dessa forma, é imprescindível ressaltar que os métodos utilizados na investigação diagnóstica da DC são a pesquisa de marcadores imunológicos/sorológicos e a biópsia endoscópica da mucosa duodenal e jejunal, estando a ultrassonografia indicada apenas no esclarecimento diagnóstico de casos suspeitos que apresentam sintomas abdominais (SILVA ED e SILVA GL, 2014). Nota-se que a triagem anual para doença celíaca com anticorpos da transglutaminase tecidual (tTGA) começou aos 2 anos de idade usando ensaios de ligação de radioligando (AGARDH DN, et al., 2017).

É válido ressaltar que os marcadores sorológicos para doença celíaca não descartam o exame histopatologiDeve-se enfatizar que, até o momento atual, os marcadores sorológicos para doença celíaca não anulam o exame histopatológico do intestino delgado, visto que, este continua sendo o padrão-ouro para o diagnóstico definitivo da doença celíaca. Contudo, a biópsia intestinal deve ser recomendada mesmo com sorologia negativa nos casos de deficiência de IgA e quando houver suspeita clínica, como nos parentes de primeiro grau de doentes celíacos com sinais ou sintomas compatíveis, uma vez que a história familiar é fundamental para suspeita diagnóstica (LUDVIGSSON JF, et al., 2014).

O teste de HLA também deve ser considerado durante uma avaliação para DC. Sabe-se que $95 \%$ dos portadores de DC são HLA DQ2 positivos e o restante é HLA DQ8 positivo. É importante salientar que os níveis de IgA total devem sempre ser solicitados para avaliar a deficiência seletiva de $\operatorname{lgA}$, porque é a deficiência imunológica mais comum em crianças. No entanto, se um indivíduo realmente tem deficiência seletiva de $\operatorname{lgA}$, o teste sorológico oferece pouca vantagem em relação ao procedimento direto para a biópsia intestinal para estabelecer o diagnóstico (WATKINS RN e ZAWAHIR SM, 2017).

\section{Tratamento}

O tratamento mais indicado para a DC é a eliminação do glúten da dieta, que é o responsável por causar a inflamação, porém com consequente restrição das escolhas alimentares. Essa restrição alimentar, por sua vez, é uma brusca mudança na rotina dos pacientes, pois o glúten está enraizado na dieta do brasileiro, o que pode levar a constrangimentos e dificultar o convívio social da criança e do adolescente (BRANCAGLIONI BA, et al., 2016; MONTEIRO E, et al., 2019).

Nesse sentido, a Lei 10.674/03 de 16 de maio de 2003 é um amparo, visto que tornou obrigatória a informação sobre a presença de glúten nos rótulos de produtos alimentícios comercializados e bulas, como medida preventiva e de controle da DC. No entanto, mesmo com o benefício da lei, a terapia nutricional deve ser realizada e acompanhada por profissionais da nutrição (BRANCAGLIONI BA, et al., 2016; MONTEIRO E, et al., 2019).

A mudança que o paciente com DC enfrenta não está relacionada apenas com a nutrição, mas também como essa modificação de hábitos pode influenciar na saúde mental delas. Sevinc E, et al. (2017) confirma que a DC, além de prejudicar a qualidade de vida, também gera psicopatologias nas crianças. Já o estudo realizado por Watkins RD e Zawahir S (2017) concluiu que o tratamento aumenta a ansiedade, já que a criança precisa estar vigilante para evitar ingerir alimentos com glúten. Por isso é necessário um acompanhamento multiprofissional, psiquiátrico, nutricional e do gastroenterologista, para obter adesão bemsucedida a dieta e consequentemente, uma qualidade de vida e saúde mental às crianças diagnosticadas com DC (CAMPOS CP, et al., 2018).

\section{CONSIDERAÇÕES FINAIS}

Diante dos fatos supracitados, este estudo reforçou que a doença celíaca é uma enteropatia crônica frequente na população mundial, embora seja subnotificada principalmente na infância. Na criança, os sintomas prevalentes na forma clássica como dor e distensão abdominal, diarreia e vômitos, ainda que considerados inespecíficos para a doença, são dignos de suspeita, já que podem repercutir sistêmica, metabólica e imunologicamente. Além disso, o diagnóstico precoce da doença celíaca faz-se importante para evitar sintomas e complicações, bem como para melhorar a qualidade de vida da criança ao longo de seu crescimento e desenvolvimento. Desse modo, a retirada do glúten da dieta ainda se configura como única abordagem terapêutica disponível, fazendo-se necessária a busca por outras formas de tratamento. 


\section{REFERÊNCIAS}

1. AGARDH DN, et al. Maternal use of dietary supplements during pregnancy is not associated with coeliac disease in the offspring: The Environmental Determinants of Diabetes in the Young (TEDDY) study. British Journal of Nutrition, 2017: 1-7.

2. BRANCAGLIONI BCA, et al. Crianças e adolescentes que convivem com diabetes e doença celíaca. Rev Gaúcha Enferm. 2016; 37(1): e53787

3. BRASIL. Ministério da Saúde. Secretaria de Atenção à Saúde. Doença Celíaca: Protocolo Clínico e Diretrizes Terapêuticas. Portaria SAS/MS no 1149. 2015.

4. CAMPOS CGP, et al. Doença celíaca e o conhecimento dos profissionais de saúde da atenção primária. R. Saúde Públ., 2018: 1(2): 54-62

5. CAVINATTO J. Doença celíaca e introdução do glúten na dieta infantil: avaliação dos riscos e benefícios. International Journal of Nutrology, 2017: 294-297.

6. CONCEIÇÂO DS, et al. Impacts of Celiac Disease on quality of life: Integrative Literature Review. International Journal of Advanced Engineering Research and Science (IJAERS), 2020; 7(5): 90-98.

7. DAMASCENO J. Associação entre doença celíaca e complicações do diabetes em pacientes com diabetes mellitus tipo 1: revisão sistemática. Rev. Soc. Bras. Clin. Med, 2017: 15 (3): 206-13

8. DIAS JA. Celiac Disease: what do we know in 2017?. Ge - Portuguese Journal Of Gastroenterology, [S.L.], 2017; 24(6): 275-278.

9. FERREIRA CT, SEIDMAN E. Food allergy: a practical update from the gastroenterological viewpoint. J Pediatr (Rio J). 2007; 83(1): 7-20.

10. IGARSABA L. Elaboration of a risk score for celiac disease screening. DEMETRA, Rio de Janeiro, 2019: 1-16.

11. ISIKAY S, KOCAMAZ H. The neurological face of celiac disease. Arq. Gastroenterol., 2015; 52(3).

12. KELLY C. Diagnosis of celiac disease. UpToDate. 2014.

13. LABRADA OB, et al. Enfermedad Celíaca en el adulto. Un reto en el nuevo milênio. Multimed. Jornal Médico. Granma, 2020; 24(4): 949-967.

14. LUDVIGSSON JF, et al. Diagnosis and management of adult coeliac disease: guidelines from the British Society of Gastroenterology, 2014; 63(8): 1210-28.

15. MARSH MN. Gluten, major histocompatibility complex, and the small intestine. A molecular and immunobiologic approach to the spectrum of gluten sensitivity ('celiac sprue'). Gastroenterology, 1992; 102(1): 330-54.

16. MONTEIRO E, et al. Deficiência de vitaminas D em crianças com doença celíaca. ACTA Portuguesa de Nutrição. 19(2019) 56-59.

17. NATIONAL INSTITUTES OF HEALTH CONSENSUS DEVELOPMENT CONFERENCE STATEMENT ON CELIAC DISEASE. Gastroenterology, 2005; 128(4 Suppl 1): S1-9.

18. NETO US, et al. Doença celíaca: características clínicas e métodos utilizados no diagnóstico de pacientes cadastrados na Associação dos Celíacos do Brasil. J Pediatr, 2001; 77 (2): 131-8.

19. OLIVEIRA GN, et al. Review of celiac disease presentation in a pediatric tertiary center. Arq. Gastroenterol., São Paulo, 2018; 55(1): 86-93.

20. OLSSON C, et al. The everyday life of adolescent coe liacs: issues of importance for compliance with the gluten-free diet. J Hum Nutr Diet., 2008; 21(4): 359-67.

21. PAULINO R. Glúten $x$ doença celíaca: uma revisão bibliográfica. Revista Campo do Saber, 2017; 13p.

22. RUBIO-TAPIA A, et al. ACG clinical guidelines: diagnosis and management of celiac disease. Am J Gastroenterol., 2013; 108(5): 656-76.

23. SDEPANIAN VL, et al. Doença celíaca: avaliação da obediência à dieta isenta de glúten e do conhecimento da doença pelos pacientes cadastrados na Associação dos Celíacos do Brasil (ACELBRA). Arq Gastroenterol., 2001; 38: 232-9.

24. SEVINÇ E, et al. Psychopathology, quality of life, and related factors in children with celiac disease. Jornal de Pediatria, [S.L.], 2017; 93(3): 267-273.

25. SILVA ED, SILVA GL. The contribution of abdominal ultrasound to diagnosis of celiac disease in children and adolescents Rev. Bras. Saúde Matern. Infant, 2014; 14(1): 47-52.

26. WATKINS RN, ZAWAHIR SM. Celiac Disease and Nonceliac Gluten Sensitivity. Pediatr Clin N Am, 2017; 64(1): 563576. 